DOI https://doi.org/10.30525/978-9934-26-007-0-13

\title{
DIE ONTOLOGIE DER AUSLEGUNG DER INTERNATIONALEN RECHTLICHEN REALITÄT
}

\author{
Karvatska S. B., Melenko S. G.
}

\section{EINFÜHRUNG}

Das moderne Völkerrecht ist mehrdimensional geworden, das Verhältnis, die Korrelation von „internationalen“ und ,inländischen“ Auslegungsobjekten haben sich erheblich verändert, im neuen internationalen Koordinatensystem wurde die Rechtsauslegung unter Beibehaltung traditioneller Modelle durchgeführt. In diesem System wird die nationale Gesetzgebung von supranationalen Körperschaften in Hinsich auf ein integratives Verständnis des Rechts und seiner Werte interpretiert. Diese Tendenzen und Veränderungen bringen Innovationen in der internationalen Rechtsordnung, dessen Umfang und deren Tiefe eine Reihe konzeptioneller Fragen für die Wissenschaft des Völkerrechts aufwerfen, die eine umfassende Grundlagenforschung erfordern. Die Wirksamkeit moderner internationalen Rechtsordnung hängt weitgehend von dem Verstehen der Erfassung und der funktionalen Rolle der Auslegungspraxis als eine Aktivität zur Festlegung des Inhalts und der Form der internationalen Rechtsrealität, die in Verbindung mit der gesammelten Auslegungserfahrung in Betracht gezogen werden soll, ab.

Das Völkerrecht ist nach seinem wesentlichen Inhalt unter den Bedingunden der Rechtsstaatlichkeit „das Recht des Gerichts“. Der Prozess der evolutionären Entwicklung von law of nature zu law of court folgte immer die Zustimmung der direkten Teilnehmer der internationalen Zusammenarbeit. Das moderne Völkerrecht, das als „Gerichtsrecht“ eine neue Qualität erlangt, wird zum Gesetz der Weltgemeinschaft und geht über die eigentlichen positivistischen Grundlagen hinaus, kombinierend Elemente des objektiven Rechtshandelns in seinen Kategorien wie Wahrheit und Gerechtigkeit. Es ist dieses Völkerrecht, das es zu einem wirksamen Mittel zur rechtlichen Unterstützung der fortschreitenden Bewegung der menschlichen Zivilisation auf dem Weg des Fortschritts macht.

Es ist keine Übertreibung zu sagen, dass das Problem der Auslegung des Völkerrechts zur Liste der „ewigen“ Probleme der Lehre und der Praxis des Völkerrechts gehört. Die Notwendigkeit einer facettenreichen konzeptuellen und rechtlichen Untersuchung dieses Rechtsphänomens wird in Zeiten radikaler systemischer Transformationen im Bereich des Völkerrechts weiter verwirklicht. 


\section{Wesentliche Elemente der Grundlagen der Rechtsauslegung im Völkerrecht}

Die neuesten Forschungsergebnisse überzeugen uns davon, dass theoretische und methodische Grundlage der Auslegung in der heimischen Doktrin des Völkerrechts noch bis zu einem gewissen Grad kritisch unaussprechlich und sogar argumentativ widersprüchlich bleibt. Die wissenschaftliche Berichterstattung über die angegebenen Probleme tendiert zur Segmentierung oder zur Einzeiligkeit des Forschungsobjekts, und der interpretative Diskurs als Ganzes ist eine unzulässige Menge der Meinungsverschiedenheiten gewohnlich. Der Kern des betrachteten Problems liegt also im mangelnden Konsens über das Verständnis der Auslegung im Völkerrecht. Übrigens das bedeutet jedoch keineswegs, dass das Spektrum von dem Forschungsinteresse begrenzt ist, sondern provoziert im Gegenteil aktive Diskussionen in der Doktrin des Völkerrechts. Konkrete Entwicklungen auf dem Gebiet der Auslegung des Völkerrechts haben trotz des wissenschaftlichen Werts und der praktischen Bedeutung immer noch keine konzeptionelle Einheit und bilden kein ganzheitliches Bild der Verwendung der Theorie der Auslegung des Völkerrechts. Und das leugnet oder widerlegt keineswegs, sondern stärkt und aktualisiert nur die Notwendigkeit, die wesentlichen Elemente der Grundlagen der Problematik der richtigen Auslegung zu identifizieren.

Die Untersuchung der wichtigsten Ansätze (Schulen) zur Auslegung internationaler Rechtsnormen ist ein notwendiger Bestandteil der Entwicklung einer allgemeinen Auslegungstheorie als Bestandteil des Auslegungsprozesses im Völkerrecht und der praktischen Umsetzung internationaler Rechtsnormen im Allgemeinen. Zweitens ermöglichen die oben genannten Ansätze (Schulen) der Rechtsauslegung das Wesen des Prozesses der Auslegung von Normen im Völkerrecht besser zu verstehen und die Mittel und die Wege zur Verbesserung der Auslegung internationaler Rechtsnormen zu ermitteln. Drittens, habend ihre Vorteile, schwächen die oben genannten Ansätze (Schulen) zur Auslegung internationaler Rechtsnormen jedoch ihre Positionen mit bestimmten Nachteilen: Befürworter statischer Auslegungstheorien berücksichtigen nicht die Veränderlichkeit der Umstände, die das Wesen und den Inhalt internationaler Rechtsnormen beeinflussen; die evolutionäre (dynamische) Auslegung ,riskiert“ von den unangemessenen Änderungen des Inhalts einer Rechtsnorm beeinflusst zu sein. Viertens, bevorzugen die Befürworter der einzelnen Ansätze (Schulen) eine bestimmte Auslegungsmethode und unterschätzen häufig die Bedeutung anderer Verfahren, dennoch erfordert eine wirksame und ordnungsgemäße Auslegung im Völkerrecht jedoch die Verwendung verschiedener Auslegungsmethoden in Kombination.

In der Doktrin des Völkerrechts nimmt das Problem der Abgrenzung der Auslegung durch Subjekte einen wichtigen Platz ein. Insgesamt unterscheidet die 
internationale Rechtswissenschaf authentische und doktrinäre Auslegung. Die normative Auslegung betrifft den allgemeinen Inhalt einer internationalen Rechtsnorm und alle Fälle ihrer Anwendung. Die kasuelle Auslegung verdeutlicht den Inhalt einer internationalen Rechtsnorm in einem bestimmten Fall.

Das maßgeblichste in der Fachliteratur ist die authentische Auslegung, die von den Parteien eines internationalen Vertrags selbst durchgeführt wird. Die Ergebnisse einer solchen Auslegung haben die gleiche Wirkung wie die internationale Rechtsnorm selbst; sie sind in einer klaren formalen Form (Vertrag, Erklärung, Kommuniqué) dargelegt oder spiegeln sich in der vereinbarten Praxis wider. Die authentische Auslegung kann Änderungen eines internationalen Vertrags enthalten und in jeder Form durchgeführt werden: Sondervertrag, Vereinbarung, Austausch von Notizen (Briefen), Protokoll und dergleichen ${ }^{1}$. Eine authentische Intrepretation ist die effektivste für die Erhaltung der Wirksamkeit eines internationalen Vertrags. Diese Auslegung hat eine lange Geschichte. Das Konzept der ,authentischen Auslegung“ ist kein Neologismus der Rechtsdoktrin des 20. Jahrhunderts. Ihre Ursprünge führen auf römische Rechtsgrundsätze, die legte die Grundlage von dem kontinentaleuropäischen Rechtssystem insbesondere beinflussten sie das preußisch und das Nachkriegsdeutsche Rechtssystem, zurück $^{2}$. Aus den Rechtssystemen einzelner Staaten verbreitete sich die authentische Auslegung allmählich auf den Bereich des Völkerrechts ${ }^{3}$.

Die authentische Auslegung sich verwirklicht von den Vertragsparteien selbst oder von einer internationale Organisation. Wichtige Subjekte dieser Form der Auslegung sind Internationaler Gerichtshof, internationale Arbitragen, verschiedene zwischenstaatliche (zwischenstaatliche) Ausschüsse, Kommissionen und dergleichen. Oft enthalten internationale Verträge selbst eine Bestimmung, dass ein Gericht oder ein Arbitrage im Falle eines Streits über das Verständnis der Bestimmungen ein Vertrag interpretieren wird. Das sind die internationalen Justizinstitutionen, die im Prozess der Rechtsauslegung eine besondere Rolle spielen. G. I. Hernández sagt dass Das Konzept der ,authentischen Auslegung“" erstmals in der Präzedenzfallspraxis des IGH der 40er Jahre erwähnt wurde ${ }^{4}$,: Richter Hudson hielt es für notwendig, „die ausdrücklichen Absichten der Parteien zu verwirklichen “5.

\footnotetext{
${ }^{1}$ Буроменський М.В. Тлумачення міжнародних договорів. Міжнародне публічне право : підручник : у 2 т. Харків : Право, 2019. Т. 1. С. 43.

${ }^{2}$ Berner K. Authentic Interpretation in Public International Law. Heidelberg Journal of International Law. 2016. Vol. 76. № 4. P. 852.

${ }^{3}$ Ibid. P. 857-858.

${ }^{4}$ Hernandez G. I. Interpretative authority and the international judiciary. Interpretation in international law / eds.: A. Bianchi, D. Peat, M. Windsor. Oxford: Oxford University Press, 2015. P. 166-188.

${ }^{5}$ Spiermann O. International Legal Argument in the Permanent Court of International Justice: The Rise of the International Judiciary. Cambridge: Cambridge University Press, 2005. P. 236-237.
} 
Besonders wichtig ist die rechtliche Auslegung von den internationalen Organisationen in Bezug auf ihre eigenen Chartas: sie ermöglicht diese Gesetze an neue Bedingungen anzupassen. Diesbezüglich ist bezeichnend die Annahme von der UN-Generalversammlung die Erklärung über Grundsätze des Völkerrechts betreffend freundschaftliche Beziehungen und Zusammenarbeit zwischen den Staaten im Einklang mit der Charta der Vereinten Nationen. Formal war das ein Akt der Auslegung der Grundsätze der Charta, aber tatsächlich das wer der wichtigste Schritt nach der Annahme der Charta in der Entwicklung des Inhalts der Grundlagen des Völkerrechts. Die Bestimmungen dieser Erklärung sind aufgrund Ihre weitere Anerkennung von den Staaten rechtsgültig geworden. Die rechtliche Auslegung der Aktivitäten internationaler Organisationen führt neue wichtige Punkte in die Funktionsweise des Völkerrechts ein. Die verabschiedeten Rechtsakte fördern das Erreichen von einheitlichem Verständnis des Inhalts internationaler Rechtsnormen. Die Auslegung wird laufend und regelmäßig unter Beteiligung einer großen Anzahl von Staaten durchgeführt. Die Ergebnisse sind mit der Autorität sowohl der Teilnehmerstaaten als auch der internationalen Organisation selbst ausgestattet. Daher kann man in der Tat über eine neue Form der Auslegung sprechen, die eine große Macht hat, das Völkerrecht zu beeinflussen.

Katharine Berner, Doktor der Rechtswissenschaften an der HumboldtUniversität und Richterin am Kammergericht, befasst sich mit der Frage der Definition von Auslegungsarten und betont die rechtlich wissenschaftliche und authentische Auslegung ${ }^{6}$. Eine authentische Auslegung in der allgemeinen Rechtstheorie ist jedoch, wie K. Berner bemerkt, nicht gleichbedeutend mit einer authentischen Auslegung im Verständnis des Völkerrechts. Der allgemeine theoretische Ansatz vermutet, dass eine Rechtsnorm von denjenigen interpretiert werden kann, die an der Gesetzgebung beteiligt sind: nur der, der die Norm erstellt hat, kann ihre endgültige „Auslegung“ erstellen - tatsächlich eine neue Norm. Alle anderen wenden die Auslegung dieser Rechtsnorm an, sie haben nicht das Recht, mit ihrer Auslegung die gleiche Rechtskraft zu verleihen, die die ursprüngliche Norm hat. Für diese anderen Ausleger schränkt das „Original“ der Norm ihre Auslegungsaktivitäten ein, und die daraus resultierenden Auslegungen sind den Gesetzgebungsakten hierarchisch untergeordnet ${ }^{7}$.

K. Berner verteidigt die Ansicht, dass internationale gerichtliche und quasigerichtlichy Behörden - der IGH, der UN-Menschenrechtsausschuss der Vereinten Nationen und andere übernehmen in gewisser Weise Rechtsetzungsfunktionen, durchführend die Lösung der Streiten, abgebend beratende Stellungnahme und veröffentlichend allgemeine Kommentare. Die vereinbarten Parteien können das Recht auf Auslegung an andere delegieren, und solche endgültigen Auslegungen

\footnotetext{
${ }^{6}$ Berner K. Op. cit. P. 857-858.

${ }^{7}$ Ibid. P. 851-852.
} 
können nach Ansicht von K. Berner als neue Rechtsquelle angesehen werden ${ }^{8}$. Unserer Meinung nach spiegelt diese Position alle Komplexitäten des methodischen Diskurses über das Problem der Gesetzgebung internationaler Justizbehörden. Tatsächlich bestätigt die Forschung, dass internationale Gerichte im Prozess der Auslegungstätigkeit Rechtsnormen interpretieren und keine neuen schaffen können. Obwohl im Falle der Schließung der Lücke in der gesetzlichen Regulierung gerichtliche Handlungen von internationalen Justizbehörden als Rechtsquelle angesehen werden können. Gleichzeitig soll man die Verwarnung von G. Lauterpacht nicht vergessen, dass ,weder die Rechtstheorie noch die politische Vorsicht die kreative richterliche Tätigkeit und die Freiheit der gerichtlichen Beurteilung abschaffen können. Der freiwillige Charakter von internationalem Gerichtsverfahren erfordert jedoch, dass der subjektive Aspekt der gerichtlichen Tätigkeit durch eine Erweiterung der Grundlagen der gerichtlichen Argumentation ausgeglichen wird"“9 .

Unterstützend diese Thesen, stellen die rumänischen Forscher Vasilica Negrut, Jana Maftei und Varvara.- L. Coman in einer wissenschaftlichen Aufsatz fest, dass die authentische Auslegung kollektiv oder einseitig sein kann. Einerseits wird die Auslegung von allen Vertragsparteien (zum Zeitpunkt der Annahme des Textes oder später) durchgeführt, andererseits kann jede Vertragspartei eigene Auslegung verwirklichen und argumentieren, wie sie ihren Text versteht ${ }^{10}$. Die Frage des kollektiven Subjekts wird auch von A. Orakhelashvili berücksichtigt : Nach Ansicht des Wissenschaftlers bedingt das „kollektive Subjekt" die Möglichkeit einer objektiven Auslegung ${ }^{11}$.

Die doktrinale Auslegung wird von Fachleuten für wissenschaftliche Arbeiten durchgeführt. Diese Art der Auslegung ist nicht rechtsverbindlich, nicht authentisch, aber das kann jedoch die Schaffung und die Anwendung des Völkerrechts beeinflussen. Je nach Thema kann die Auslegung objektiv sein, wobei nur der eigentliche Text eines internationalen Vertrags berücksichtigt wird, und subjektiv, wobei in diesem Prozess nach dem wahren Willen der Parteien eines internationalen Vertrags gesucht wird.

Es ist beachtenswert, dass in den letzten Jahren das Interesse an den Problemen der doktrinären Rechtsauslegung in der Gemeinschaft der ukrainischen theoretischen Wissenschaftler spürbar gestiegen ist. Wie jedoch von M. Sadovskiy festgestellt wurde, fehlt der Ukraine immer noch eine umfassende Untersuchung des Problems der Prinzipien der doktrinären Auslegung des Rechts ${ }^{12}$.

\footnotetext{
${ }^{8}$ Berner K. Op. cit. P. 852.

${ }^{9}$ Lauterpacht H. Some Observations on Preparatory Work in the Interpretation of Treaties. Harvard Law Review. 1935. Vol. 48, Iss. 4 (Febr.). P. 575.

${ }^{10}$ Maftei J, Coman V. L. Interpretation of Treaties. Acta Universitatis Danubius: juridica. 2012. Vol. 8. No. 2. P. 21.

${ }^{11}$ Orakhelashvili A. The Interpretation of Acts and Rules in Public International Law. P. 286.

12 Садовський М. М. Загальна характеристика універсальних принципів доктринального тлумачення права. Науковий вісник Міжнародного гуманітарного університету. Серія: Юриспруденщія. 2015. № 15. Т. 1. С. 44.
} 


\section{Das Problem der Definition des Auslegungsobjekt im Völkerrecht}

Bei der Auswahl einer methodischen Grundlage muss der Interpreter bestimmen, was Gegenstand seiner Tätigkeit ist. Eine klare Definition des Auslegungsobjekts ist äußerst wichtig, da das Auslegungsobjekt und die Auslegungsmethoden miteinander zusammenhängen ${ }^{13}$. Als Bestätigung kann man die abweichende Meinung betreffs Gerichtsprozess zum Thema von den Land-, Insel- und Seegrenzen vom IGH-Richter T. Bernardese als Beispiel nennen. In dieser Meinung hat Herr Bernardese festgestellt dass die Auslegung, die (aus praktischen Gründen) den Gegenstand und den Zweck des Vertrags oder die zu interpretierende Begriffe oder Ausdrücke ablehnt, kann als die Auslegung nich genannt $\operatorname{sein}^{14}$. Aufbauend auf diesen methodischen Vorschlag David S. Jonas, ein amerikanischer Anwalt, Partner bei «Fluet, Huber \& Hoang» und Thomas N. Saunders, ein stellvertretender Staatsanwalt der Vereinigten Staaten in Washington, D.C. begründete die Abhängigkeit der Auslegung von „Objekt und

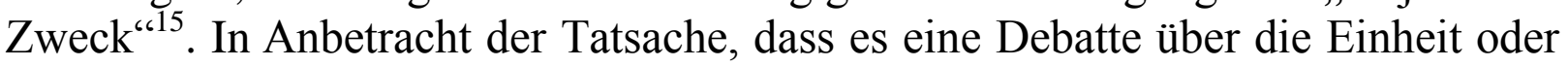
die Differenzierung der Begriffe „Objekt und Zweck“ gibt, neigen die Autoren dazu zu denken, dass diese beiden Begriffe als ein einziges Konzept betrachtet werden sollten, da diese Unterscheidung nicht praktisch ist, sondern verkompliziert es die Auslegung ${ }^{16}$.

Aufgrund der Unsicherheit, die mit dem kontroversen Charakter der Frage des Objekts und des Subjekts der Auslegung verbunden ist, gibt es unter Forschern (sowohl Rechtstheoretiker als auch Völkerrechtler) keine Einheit hinsichtlich der Institutionalisierung des Subjects und des Themas der Auslegung und der Auslegung des Rechts im Völkerrecht.

Das Subjekt der Auslegungstätigkeit ist der Text eines Rechtsakts, und sein Objekt sind Rechtsregeln. Das endliche Ziel der Rechtsauslegung besteht darin, die Bedeutung aufzudecken und den Inhalt der entsprechenden Verhaltensregeln zu klären. Das ist ein Versuch, das Verständnis der Auslegung über die positivistische Vision hinaus zu bringen.

Im Völkerrecht ist es noch offensichtlicher, dass die Rechtsauslegung keine Kategorie der Doktrin, sondern der Praxis ist, deren Subjekt der Umsetzung gerichtliche und gerichtsähnliche Organe und Institutionen sind. Das endliche Ziel der Rechtsauslegung im Allgemeinen und des Völkerrechts im Besonderen besteht nicht darin, den Inhalt der entsprechenden abstrakten Verhaltensregeln

\footnotetext{
${ }^{13}$ Karvatska S. Object and means as interpretation key factors in international law. Visegrad Journal on Human Rights. 2018. № 5 (Vol. 2). P. 37-42.

${ }^{14}$ Land, Island and Maritime Frontier Dispute (El Salvador v. Honduras: Nicaragua intervening). Judgment of 11 September 1992 / ICJ Reports. 1992. P. 383. Para 206.

15 Jonas David S., Saunders Thomas N. The Object and Purpose of a Treaty: Three Interpretive Methods. P. 565-609.

${ }^{16}$ Ibid. P. 580.
} 
(Rechtsnormen) zu klären. Das Hauptmerkmal der Auslegung im Völkerrecht drückt sich darin aus, dass sie nicht nur als Akt der Erkenntnis (Klärung) des Inhalts einer Rechtsnorm in ihrer äußeren Form - im Texte eines internationalen Rechtsakts (Vertrag, Charta, vorbereitende Materialien, Beschluss) - sondern auch erscheint in gewissem Sinne ein Akt der Modellierung des Rechtes. Das Konzept der „Rechtsauslegung im weitesten Sinne“ spiegelt also sowohl den Prozess als auch das Ergebnis wider.

Wir bestehen auf diesem Begriff der Auslegung im weitesten und im engsten Sinne und halten es für notwendig, unsere Argumentation mit dem konzeptuellen Argument von A. Barak zu verstärken. In einem seiner modernsten und systemischsten Werke, das sich mit dem Problem der Rechtsauslegung befasst «Purposive Interpretation in Law» ${ }^{17}$ - stellt er fest, dass ein Text zu interpretieren bedeutet seiner rechtlichen Bedeutung aus einer Reihe semantischer Möglichkeiten wählen und welche der semantischen Bedeutungen des Textes seine eigentliche rechtliche Bedeutung darstellt entscheiden ${ }^{18}$. Darüber hinaus ist jedes Verhalten zum Zweck der Auslegung „Text" ${ }^{\text {"19 }}$.

Davon überzeugen auch die neuesten Studien einheimischer Wissenschaftler: N. Guralenko merkt an, dass „die werteorientierte Rechtsposition, nach der beschränkt sich die Rechtsauslegung nicht auf die Suche vom Sinn eingeführt von dem Gesetzgeber, sondern ist das ein Akt der" kreativen Rechtsetzung ist, überwiegt. Der Wissenschaftler behauptet dass ,in ihrer wert-semantischen Dimension die Auslegung ist zweiwertig : das ist nicht nur ein Akt der Erkenntnis, die Festsetzung was bereits Rechtskraft hat, sondern auch ein Akt der Schaffung eines neuen Gesetzes ${ }^{\text {“20. }}$.

Unserer Meinung nach, hängt im Allgemeinen die Definition der Auslegung und das Verständnis ihres Wesens von den methodischen Einstellungen, an denen derjenige festhält, der die Definition gibt, sowie von dem Sinn, in dem wir über die Auslegung im Völkerrecht sprechen werden - im engeren oder im weiten Sinne, ab. Zum Beispiel erlaubt das erste Einfahren, das den Text als hauptsächlich definiert und die Auslegung als Sinnfindung postuliert, existiert direkt im Text, erlaubt uns die folgende Definition zu geben: „Die Aufgabe der Auslegung zielt darauf $a b$, die genaue Bedeutung und den Inhalt der Rechtsstaatlichkeit zu bestimmen, die in einer bestimmten Situation angewendet wird“. Das zweite quasi-textuelle Einfahren berücksichtigt die Auslegung des „Lichts des Textes“ (à la lumière du texte): „Die vertragliche Auslegung zielt darauf ab, die wahre Bedeutung des Vertrags festzustellen“. Das dritte, subjektive Einfahren schreibt dem Text, der als Mittel zum Verständnis der Absichten der

\footnotetext{
${ }^{17}$ Barak A. Purposive Interpretation in Law / transl. from the Hebrew by Sari Bash. P. 3-4.

${ }^{18}$ Ibid.

${ }^{19}$ Ibid.

${ }^{20}$ Гураленко Н.А. Суддівське правопізнання: праксеологічний вимір : монографія. С. 298.
} 
Vertragsparteien angesehen wird, große Bedeutung zu: „Auslegung ist der Prozess der Bestimmung des rechtlichen Charakters und der Konsequenzen von den Parteien erzielten Konsenses zur Ausführung der erklärten Absicht. <..> Diese Absicht wird in Worten unter bestimmten Umständen ausgedrückt ${ }^{\star 21}$. Das Spektrum der praktischen Füllung des Problems der Auslegungsansätze ist ziemlich breit: Dem Text wurde immer eine entscheidende Bedeutung beigemessen (die Auslegung, die den Text nivelliert, ist ebenfalls ungebührlich), und aus einem anderen Blickwinkel ist es sinnlos, andere Auslegungsmittel zu ignorieren (wer die Buchstaben hält, hält dann die Oberfläche).

Die Textualisten bekennen der wörtliche Text eines internationalen Rechtsakts als das einzige Auslegungsobjekt. Zur Unterstützung ihrer Position argumentieren Befürworter dieses Ansatzes, dass die Absichten der Parteien, die sich nicht im Text widerspiegeln, keine Rolle spielen. Sie berücksichtigen jedoch nicht die Tatsache, dass sich die ursprüngliche Absicht im gegenseitigen Einvernehmen der Parteien eines internationalen Vertrags ändern kann.

Laut A. Orakelashvili hängt die Auslegung im Völkerrecht nicht von Regeln und Methoden (Methoden) der Auslegung, die im nationalen Recht angewendet werden können, sondern auch nicht von der Gesetzgebung eines bestimmten Landes ab. Der Autor ist sich jedoch sicher, dass es bestimmte Auslegungsgrenzen gibt: Im Vertrag selbst sind jedenfalls klare Anforderungen an die Auslegung festgelegt ${ }^{22}$. Im Kontext des Verhältnisses zwischen positivem und natürlichem Recht verteidigt $\mathrm{A}$. Orakhelashvili die Bestimmtheit des rechtlichen Inhalts der Normen und die Objektivität ihrer Auslegung im Gegensatz zum Subjektivismus, insbesondere aufgrund der Ursache der Manipulation des Rechts. Er ist der Ansicht, dass die Frage der Rechtmäßigkeit der Handlung eines bestimmten Gegenstandes des Völkerrechts ausschließlich auf der Grundlage des positiven Rechts entschieden werden sollte. Wenn die Maßnahme definitiv über den Rahmen des positiven Rechts hinausgeht, muss sie mit dem Naturrecht vereinbar sein, in der Lage sein, mehr Fragen zu beantworten und solche Grundsätze wie Integrität, Gleichheit der Staaten usw. zu berücksichtigen. Das Hauptziel der Auslegung des Völkerrechts ist nach der meinung von A. Orakhelashvili die Einführung von der Sicherheit, von der Vorhersehbarkeit und von der Kohärenz in die internationale Rechtsordnung. A. Orakhelashvili lehnt den soziologischen Ansatz bei der Auslegung des Völkerrechts kategorisch $a b$, wenn ein solcher Ansatz die Hierarchie, die in dem Wiener Übereinkommen über das Recht der Verträgeveran befestigt ist, leugnet. Der Wissenschaftler betont auch die Notwendigkeit einer Auslegung, die frei von dem Einfluss

\footnotetext{
${ }^{21}$ Lévesque J.-Fr. Traités de verre : Réflexions sur l'interprétation. Revue québécoise de droit international. 2006. Vol. 19. № 1. P. 68.

${ }^{22}$ Orakhelashvili A. The Interpretation of Acts and Rules in Public International Law. P. 286.
} 
politischer Faktoren auf die Festlegung des Inhalts von den Normen, die von den Parteien (Staaten) vereinbart sind.

Im Rahmen der Diskussion über das Objekt der Auslegung im Völkerrecht ist die französische Anwaltin für EU-Recht I. Van Damme eher der Ansicht, dass die Auslegung kontextabhängige ist, und betont, dass das Vorrecht des Interprets darin besteht, zu entscheiden, wie der Kontext in Kraft tritt. In ähnlicher Weise erklärt der britische Forscher R. Gardiner (University College London), dass die gewöhnliche Bedeutung nicht aus dem Kontext herausgenommen werden kann, da sie direkt und eng mit dem Kontext verbunden ist und zusammen mit allen anderen relevanten Elementen von WÜRV genommen werden muss. Die Position von W. Linderfalk, Professor an der Rechtsfakultät der Universität Lund (Schweden), scheint nicht eindeutig zu sein: Er nimmt eine „durchschnittliche“ Position zwischen radikalem Rechtsskepsis ein, die die Auslegung eines Vertrags als eine Kunst betrachtet, die nicht durch Regeln adjustiert werden kann (Auslegung der Meinuch vom Autor nach ist eine politische Aktivität) und eine Position, die anerkennt, dass der Interpret Auslegungsregeln anwendet, um mit neutralen Mitteln ein Ergebnis zu erzielen. Der Auslegungsprozess wird, wie V. Linderfalk feststellt, von einem Regelsystem, das einerseits die politischen Erwägungen erheblich einschränkt und andererseits aufgrund einer begrenzten Anzahl von Grundregeln dem Interpret große Manövriermöglichkeiten beistellt, reguliert ${ }^{23}$.

Die strukturelle Grundlage der Arbeit „Interpretation in International Law“ $(2015)^{24}$ ist eine Metapher des Spiels, die die Komponenten der Auslegungsaktivität verdeutlicht, insbesondere die Rhetorik in der Auslegung, die Gebundenheit der Auslegungsaktivitäten internationaler Institutionen, die Organisation und Fachleute zu ethischen Postulaten und die Verbindung der Auslegung mit der Politik.

\section{Grundsätze der Rechtsauslegung als grundlegende Ideologie des Rechtsauslegungsprozesses}

Aus objektiven Gründen verwendet man bei der Auslegung verschiedene Rechtssysteme und unterschiedliche Auslegungsprinzipien als Methodologie. Gleichzeitig kann man die Tendenz, sie zu vereinheitlichen, seit langem verfolgen, obwohl dieser Prozess von ernsthaften Diskussionen zwischen Theoretikern und Praktikern über die „richtige“, „objektive“ einheitliche Interpretationsmethodologie begleitet wird. Dieses Problem ist im Völkerrecht besonders akut geworden. Das sind die Spezialisten des Völkerrechts, die heute Leader in den interpretativen und methodischen Debatten sind. Das erklärt mehrere gute Faktoren: eine bedeutende politische Komponente internationaler Rechtsprozesse, die Dynamik des Systems

\footnotetext{
${ }^{23}$ Waibel M. Demystifying the Art of Interpretation.. P. 576.

${ }^{24}$ Bianchi A., Peat D., Windsor M. (eds.). Interpretation in international law. Oxford : Oxford University Press, 2015. 399 p.
} 
des Völkerrechts, das Vorhandensein mehrerer mächtiger, langjähriger Rechtstraditionen (insbesondere angelsächsischer und kontinentaler) und das Fehlen einer einheitlichen Auslegungsmethodologie.

Die Prinzipien der Rechtsauslegung sind die grundlegenden axiomatischem Grundlage von dem Rechtserkenntisprozess und von dem Rechtsauslegungsprozess. Die Grundsätze der Rechtsauslegung bestimmen in der Tat die unbestreitbaren Grundsätze, von denen sich der Interpret leiten lassen soll, um den wahren Inhalt einer Rechtsvorschrift $\mathrm{zu}$ verstehen und letztlich ihre wirksame Umsetzung sicherzustellen. Im Gegensatz zu den Grundnormen haben die Interpretationsprinzipien besondere Funktionen. Vor allem wirken sie der Fragmentierung des Völkerrechts entgegen und sorgen auch für eine gewisse Objektivität der Entscheidungen internationaler Gerichte. Die Auslegungsgrundsätze garantieren erstens die Autonomie des Rechtssystems; zweitens tragen sie zur Stabilität und zur Zuverlässigkeit des Rechtssystems bei; drittens stärken sie die Einheit und die Integrität des Rechtssystems: beseitigen die Konflikten zwischen verschiedenen Primärnormen; Viertens modellieren sie Methoden, um Lücken im Rechtsrahmen zu schließen und die Ausübung der richterlichen Gewalt einzuschränken, indem sie Auslegungsgrenzen festlegen ${ }^{25}$.

In der Geschichte der Rechtsprechung gab es viele Versuche, universelle Auslegungsprinzipien zu entwickeln. Mitte des letzten Jahrhunderts formulierte der italienische Philosoph Emilio Betty in seinem berühmten Werk „Hermeneutik als allgemeine Methodik der Geisteswissenschaften" die Auslegungsprinzipien, die er hermeneutische Kanone nannte. Die ersten beiden dieser Kanons betrafen das Objekt, die anderen das Subjekt. Der erste Kanon ist der Kanon der hermeneutischen Autonomie eines Objekts (oder der Kanon der Immanenz des hermeneutischen Maßstab), der zweite ist der Kanon der hermeneutischen Gesamtheit oder Integrität, der dritte ist der Kanon der Relevanz des Verstehens, der vierte ist der Kanon der semantischen Korrespondenz.

Das Wiener Übereinkommen über das Recht der Verträge von 1969 befestigte die Grundprinzipien der Auslegung der Normen internationaler Verträge, die bis heute gültig sind, und die ermöglichen die Entwicklung der Methodik der Auslegungspraxis.

Die Grundsätze und die Regeln für die Auslegung internationaler Verträge werden hauptsächlich von dem WÜRV kodifiziert (Artikel 31 und 32). Nach Ansicht dem IGH können verkörperte in diesen Artikeln Grundsätze ,in vielerlei Hinsicht als Kodifizierung der bestehenden Regeln des Völkergewohnheitsrechts angesehen werden“.

\footnotetext{
${ }^{25}$ Aust H. Ph, Rodiles A., Staubach P. Unity or Uniformity? Domestic Courts and Treaty Interpretation. Leiden Journal of International Law. 2014. Vol. 27. P. 79.
} 
Nach der Position von M. Weibel sind für die von WÜRV definierten Prinzipien did Logik und eind bestimmten Ordnung, die die Fähigkeiten des Interprets gleichzeitig einschränken und erweitern, inhärent ${ }^{26}$. Nebenbei stellen wir fest, dass die Annahme von WÜRV, wie in den Studien ausländischer Autoren hervorgehoben wird, viele Interpretationsprobleme „beseitigt" hat. So spricht insbesondere A. Orakelashvili über die dreifachen Auswirkungen dieser Einwikrung auf das Völkerrecht. Nach Ansicht des Autors formulierte der WÜRV schließlich ein einheitliches Interpretationsregime auf der Grundlage von Regeln, das keinen Raum für Meinungsverschiedenheiten ließ (hauptsächlich zwischen der Vision des Interpretationsprozesses durch verschiedene Schulen). Zweitens hat die WÜRV die Unterscheidung zwischen der Allgemeinen Auslegungsregel (Art. 31) und zusätzlichen Auslegungsmethoden (Art. 32) gefestigt, die nicht alternativ und autonom werden können. Drittens lässt WÜRV nicht zu, dass die Absichten von Staaten als unabhängiger und autonomer Interpretationsfaktor angesehen werden ${ }^{27}$. Nach unserer Auffassung bleiben die von WÜRV festgelegten Auslegungsregeln bis heute relevant, müssen jedoch selbst bereits nach modernen Gegebenheiten angepasst werden.

Unter den Grundsätzen der Auslegung im Völkerrecht wird die Sonderbedeutung von der Gewissenhaftigkeit besondere Bedeutung beigemessen (Auslegung muss gewissenhaft und ehrlich sein), um die Interessen aller interessierten Parteien zu berücksichtigen und den tatsächlichen Inhalt einer internationalen Rechtsnorm zu ermitteln. Laut dem Professor für Völkerrecht und internationale Beziehungen, C. Fernandez de Casadevante Romani (Madrid, Spanien), schränkt die Gewissenhaftigkeit die Ermessensfreiheit der Staaten bei der Auslegung ein und verhaltet ihre Willkürr ${ }^{28}$. In der Tat betrifft eine tiefere Ermessensfreiheit, wie G. Hristova treffend bemerkt, genau die zulässige Grenze bei der Wahl der Mittel $^{29}$. Es ist ganz natürlich anzunehmen, dass eine gewissenhafte Auslegung eines internationalen Vertrags die Entstehung zwischenstaatlicher Streitigkeiten verhindert, während eine unlautere Auslegung die Grundlage für rechtswidrige Handlungen sein kann, beispielsweise die Nichterfüllung übernommener Verpflichtungen oder deren unzulässige Erfüllung.

Das Prinzip des Einflusses politischer Moral oder ,politischer Zweckmäßigkeit“ führt $\mathrm{zu}$ kontroversen Diskussionen in der wissenschaftlichen Gemeinschaft. Einerseits sind Politik und Völkerrecht eng miteinander verbunden, andererseits waren und bleiben sie unterschiedliche Regulierungssysteme. Die Auslegung im Völkerrecht soll nicht von der politischen Moral bestimmt werden. Diese Meinung

\footnotetext{
${ }^{26}$ Waibel M. Demystifying the Art of Interpretation. P. 576.

${ }^{27}$ Orakhelashvili A. The Interpretation of Acts and Rules in Public International Law. Chap. 10.

${ }^{28}$ Fernandez de Casadevante Romani C. Sovereignty and Interpretation of International Norms .P. 234.

${ }^{29}$ Христова Г. О. Доктрина позитивних зобов'язань держави у сфері прав людини : автореф. дис. ... дра юрид. наук : 12.00.01. Харків. 2019. 42 с.
} 
wird von Gianluigi Palombella, Professor für angewandte Rechtstheorie an der Universität von Parma (Italien) unterstüzt. Er ist überzeugt dass das Prinzip der Reduzierung der souveränen Befugnisse von Staaten ,in Bezug auf verschiedene mögliche internationale Rechtsordnungen nicht ausreichend definiert ist ${ }^{\star 30}$.

Das Prinzip der Effektivität der Auslegung erfordert, dass die Ergebnisse der Auslegung zur Erreichung des Ziels einer internationalen Rechtsnorm beitragen und diese Norm wirksam machen. Nach Absicht vom IGH ist dieses Prinzip eines der Grundprinzipien der Auslegung, „das von der internationalen Justiz konsequent eingehalten wird“. Die Wiener Übereinkommen spiegelten dieses Prinzip als eine Anforderung wider, dass die Auslegung ,im Lichte des Objekts und des Zwecks des Vertrags" verwirklicht muss. Um dieses Prinzip zu verstehen, ist die Schlussfolgerung in der Entscheidung des Internationalen Strafgerichtshofs für das ehemalige Jugoslawien vom 2. Oktober 1995 bedeutungsvoll: „Innerhalb der Grenzen des geltenden Völkerrechts sollte die Charta der Vereinten Nationen so ausgelegt werden, dass die Wirksamkeit dieses Ziels sichergestellt wird ${ }^{31 \text { ، }}$.

Vor allem muss man sich vor einer solchen Auslegung hüten, die den Vertrag neutralisieren könnte oder unmöglich seine Erfüllung machte. Schließlich ist die Auslegung internationaler Verträge die erste wirkliche Phase bei der Realisation ihrer Einhaltung als Verhaltenskodex und als Standard im Streitfall ${ }^{32}$. Das Hauptaugenmerk der Auslegungstätigkeit soll die Realisation einer internationalen Rechtsnorm sein, gleichzeitig soll das Ergebnis der Auslegung in keinem Fall der Norm des Völkerrechts widersprechen.

Das Prinzip der Objektivität (Wahrhaftigkeit) kann als eines der wichtigsten Prinzipien der Auslegung im Völkerrecht angesehen werden. So unterscheidet Professor Olivier Corten, Direktor des belgischen Zentrums für Völkerrecht und Soziologie, objektive, voluntaristische und relativistische Interpretationsvisionen. Befürworter des Relativismus behaupten, dass eine objektive Auslegung grundsätzlich unmöglich ist, da sich der Interpret im klaren Rahmen des Rechtssystems befindet und daher keine Objektivität erreichen kann. Objektivisten und Voluntaristen glauben an die Objektivität der Interpretation. Der einzige Unterschied besteht darin, dass für die ersteren das Objektive ist das, was den sozialen Bedürfnissen entspricht, und für die letzteren, was dem Willen der Staaten entspricht ${ }^{33}$.

\footnotetext{
${ }^{30}$ Palombella G. International law raison d'être and the grounds for the interpretive approach. P. 166.

${ }^{31}$ Prosecutor v. Dusko Tadic aka «Dule» (Decision on the Defense Motion for Interlocutory Appeal on Jurisdiction) IT-94-1, of 2 October 1995 / International Criminal Tribunal for the former Yugoslavia (ICTY). 1995.

${ }^{32}$ Lévesque J.-Fr. Traités de verre : Réflexions sur l'interprétation. Revue québécoise de droit international. P. 53.

${ }^{33}$ Corten O. Les techniques reproduites aux Articles 31 à 33 des conventions de Vienne: approche objectiviste ou approche volontariste de l'interprétation? Revue generale de droit international public. 2011. Vol. 115 . № 2. P. 364.
} 


\section{SCHLUSSFOLGERUNGEN}

Also sind einzelne Auslegungsfragen im Völkerrecht so widersprüchlich, dass sie kein Konsensformat bilden können. Dazu gehört das Problem der Bestimmung des Auslegungsobjekt im Völkerrecht. Die Prinzipien der Rechtsauslegung im Völkerrecht sind axiomatische Grundlage für die Erkenntnis der internationalen Rechtsrealität, das ist die fundamentale „Ideologie“ des Rechtsauslegungsprozesses.

Die Integrationsprozesse in der modernen Welt setzen dringende Fragen auf die Tagesordnung, deren Lösung ohne ein koordiniertes Zusammenspiel von nationalem und internationalem Recht grundsätzlich nicht möglich ist. Das Entstehen einer qualitativ neuen internationalen Rechtsordnung begleitet von der aktiven Erscheinung neuer internationaler Subjekte von der Rechtsauslegung, die sich auf die nationalen Rechtssysteme auswirken. Die Effektivität des Funktionierens der modernen internationalen Rechtsordnung hängt in erster Linie von der funktionalen Rolle der Auslegungspraxis als Aktivität zur Feststellung des Inhalts und der Form von Rechtsphänomenen, die mit den von internationalen Institutionen und Organisationen gesammelten Auslegungserfahrungen in Einklang gebracht werden, ab. Konzeptionelle Opposition und sogar widersprüchliche Ansichten zur Interpretation standen schon immer im Mittelpunkt methodischer Debatten. Die Vielfalt der Akzente im Diskurs über die Auslegung des Völkerrechts hat jedoch ein gemeinsames Merkmal: Jeder ideologische Schwerpunkt wird weitgehend von Vorstellungen über die Kenntnis des Rechts determiniert.

Die Rechtsauslegung im Völkerrecht ist keine Kategorie der Dorktin, sondern der Praxis, deren Verwirklichung internationale gerichtliche und gerichtsähnliche Institutionen sind. Die Auslegung im Völkerrecht ist sowohl ein erkenntnistheoretischer als auch ein kreativer Prozess. Einerseits zielt die Auslegung darauf $\mathrm{ab}$, die bereits bestehende rechtliche Bedeutung eines internationalen Rechtsakts festzustellen, andererseits hat der Auslegungsprozess eine kreative Dimension. Konkrete Ausarbeitungen auf dem Gebiet der Auslegung des Völkerrechts haben trotz des wissenschaftlichen Werts und der praktischen Bedeutung immer noch keine konzeptionelle Einheit und bilden kein ganzheitliches Bild der Verwendung der Theorie der Auslegung des Völkerrechts.

\section{ZUSAMMENFASSUNG}

Der Artikel analysiert die Probleme der Ontologie der Interpretation der internationalen Rechtsrealität. Es wurde festgestellt, dass die Integrationsprozesse in der modernen Welt setzen dringende Fragen auf die Tagesordnung, deren Lösung ohne ein koordiniertes Zusammenspiel von nationalem und internationalem Recht grundsätzlich nicht möglich ist. Es ist beschlossen die Effektivität des Funktionierens der modernen internationalen Rechtsordnung hängt in erster Linie von der funktionalen Rolle der Auslegungspraxis als Aktivität zur 
Feststellung des Inhalts und der Form von Rechtsphänomenen, die mit den von internationalen Institutionen und Organisationen gesammelten Auslegungserfahrungen in Einklang gebracht werden, ab. Es ist belegt, dass die Rechtsauslegung im Völkerrecht ist keine Kategorie der Dorktin, sondern der Praxis, deren Verwirklichung internationale gerichtliche und gerichtsähnliche Institutionen sind. Die Auslegung im Völkerrecht ist sowohl ein erkenntnistheoretischer als auch ein kreativer Prozess. Die Analyse und das Verständnis der Auslegung des Völkerrechts als komplexes Rechtsphänomen sollen ein Schritt zur Steigerung der Effizienz der Interaktion zwischen Rechtswissenschaft und Rechtspraxis sein.

\section{LITERATURVERZEICHNIS}

1. Буроменський М.В. Тлумачення міжнародних договорів. Міжнародне публічне право : підручник : у 2 т. за ред. В.В. Мицика. Харків : Право, 2019. T. 1.

2. Berner K. Authentic Interpretation in Public International Law. Heidelberg Journal of International Law. 2016. Vol. 76. № 4. P. 845-878.

3. Hernandez G. I. Interpretative authority and the international judiciary. Interpretation in international law / eds.: A. Bianchi, D. Peat, M. Windsor. Oxford : Oxford University Press, 2015. P. 166-188.

4. Spiermann O. International Legal Argument in the Permanent Court of International Justice: The Rise of the International Judiciary. Cambridge : Cambridge University Press, 2005. 539 p.

5. Lauterpacht H. Some Observations on Preparatory Work in the Interpretation of Treaties. Harvard Law Review. 1935. Vol. 48, Iss. 4 (Febr.). P. 549-591.

6. Maftei J, Coman V. L. Interpretation of Treaties. Acta Universitatis Danubius: juridica. 2012. Vol. 8. No. 2. P. 16-30.

7. Orakhelashvili A. The Interpretation of Acts and Rules in Public International Law. Oxford : Oxford University Press, 2008. XXVII, 594 p.

8. Садовський M.M. Загальна характеристика універсальних принципів доктринального тлумачення права. Науковий вісник Міжнародного гуманітарного університету. 2015. № 15. Т. 1. С. 44-46.

9. Karvatska S. Object and means as interpretation key factors in international law. Visegrad Journal on Human Rights. 2018. № 5 (Vol. 2). P. 37-42.

10. Land, Island and Maritime Frontier Dispute (El Salvador v. Honduras: Nicaragua intervening). Judgment of 11 September 1992 / ICJ Reports. 1992. P. 383. Para 206.

11. Jonas David S., Saunders Thomas N. The Object and Purpose of a Treaty: Three Interpretive Methods. Vanderbilt Journal of Transnational Law. 2010. Vol. 43. № 3 (May). P. 565-609.

12. Barak A. Purposive Interpretation in Law / transl. from the Hebrew by Sari Bash. Princeton : Princeton University Press. 2005. 448 p. 
13. Гураленко Н. А. Суддівське правопізнання: праксеологічний вимір : монографія. Чернівці : Технодрук, 2013. $351 \mathrm{c.}$

14. Lévesque J.-Fr. Traités de verre: Réflexions sur l'interprétation. Revue québécoise de droit international. 2006. Vol. 19. № 1. P. 53-78.

15. Waibel M. Demystifying the Art of Interpretation. The European Journal of International Law. 2011. Vol. 22. No. 2. P. 571-588.

16. Bianchi A., Peat D., Windsor M. (eds.). Interpretation in international law. Oxford : Oxford University Press, 2015. 399 p.

17. Karvatska S. Interdisziplinäre Perspektive der Entwicklung von modernen interpretativen Ansätzen im Völkerrecht. European Journal of Humanities and Social Sciences. 2018. Iss. 3. S. 151-156.

18. Ajevski M. Interpretation and the Constraints on International Courts. MultiRights Research Paper. 2012. No. 12/15. 55 p.

19. Aust H. Ph, Rodiles A., Staubach P. Unity or Uniformity? Domestic Courts and Treaty Interpretation. Leiden Journal of International Law. 2014. Vol. 27. P. 75-112.

20. Fernández de Casadevante Romani C. Sovereignty and Interpretation of International Norms. Berlin : Springer, 2007. XI, 302 p.

21. Христова Г. О. Доктрина позитивних зобов'язань держави у сфері прав людини : автореф. дис. ... д-ра юрид. наук : 12.00.01. Харків. 2019. 42 с.

22. Palombella G. International law raison d'être and the grounds for the interpretive approach. Kutafin University Law Review. 2014. Vol. 1. Iss. 2. P. 153-179.

23. Prosecutor v. Dusko Tadic aka «Dule» (Decision on the Defense Motion for Interlocutory Appeal on Jurisdiction) IT-94-1, of 2 October 1995 / International Criminal Tribunal for the former Yugoslavia (ICTY). 1995.

24. Corten $\mathrm{O}$. Les techniques reproduites aux Articles 31 à 33 des conventions de Vienne: approche objectiviste ou approche volontariste de l'interprétation? Revue generale de droit international public. 2011. Vol. 115. № 2. P. 351-366.

Information about authors: Karvatska S. B.,

Doctor of Law, Associate Professor, Associate Professor at the Department of European Law and Comparative Law Yuriy Fedkovych Chernivtsi National University 2, Kotsyubynskoho str., Chernivtsi, 58012, Ukraine

Melenko S. G., Doctor of Law, Professor, Professor at the Department of European Law and Comparative Law Yuriy Fedkovych Chernivtsi National University 2, Kotsyubynskoho str., Chernivtsi, 58012, Ukraine 